\title{
MIOMATOSIS UTERINA
}

\author{
Dr. Alberto Zabaleta Lombana*
}

La consulta por miomatosis uterina responde a la más frecuente patología del aparto genital femenino; y en igual manera, constituye en el medio hospitalario una gran parte del trabajo diario de la unidad ginecológica. Lo anterior nos indujo a la elaboración del presente trabajo con miras a informarnos de algunos de sus aspectos, en particular, lo relativo a la aparente esterilidad que le acompaña.

Presentamos, pues, 188 casos de miomatosis uterina de la clientela ginecológica del Hospital Universitario Santa Clara, de Cartagena. Este personal residenciado en la ciudad o en localidades rurales vecinas, lo forman mujeres de clase social baja, económica y culturalmente pobres.

Para los fines del trabajo se desecharon las historias que no tenían la confirmación de anatomía patológica. En estas condiciones se estudiaron 19, 23, 38, 34, 32 y 42 casos entre 1961 y diciembre de 1966 respectivamente.

\section{Edad}

Acorde con lo señalado por todos los autores, la miomatosis es una patología con predilección clínicamente manifiesta entre la tercera y cuarta década de la vida. Si bien es cierto que su lenta formación se ha iniciado con más o menos años de anticipación, la sintomatología y por encle el motivo de consulta ocurre en la gran mayoría de las veces en los términos citados. En nuestra revisión, 154 mujeres (82.4\%) fueron intervenidas entre los 30 y 49 años de edad. (Cuadro No 1)

\section{CUADRO № 1}

\begin{tabular}{|c|c|c|c|c|c|}
\hline Grupos & de & edades & No de & casos & Porcentajes \\
\hline $23 a$ & 24 & años & & 3 & 1.6 \\
\hline $25 a$ & 29 & " & & 9 & 4.8 \\
\hline $30 a$ & 34 & " & & 20 & 10.6 \\
\hline $35 a$ & 39 & $" \prime$ & & 48 & 25.5 \\
\hline $40 \mathrm{a}$ & 44 & " & & 51 & 26.9 \\
\hline $45 \mathrm{a}$ & 49 & " & & 35 & 19 \\
\hline $50 a$ & 54 & $"$ & & 14 & 7.4 \\
\hline $55 a$ & 59 & " & & 4 & 2.1 \\
\hline \multirow{2}{*}{\multicolumn{2}{|c|}{$64-69-74-7$}} & & & 4 & 2.1 \\
\hline & & TOTAL: & 188 & casos & \\
\hline
\end{tabular}

Las pacientes de menor edad fueron tres: 2 de 24 años; una de ellas G.4 P.O A3 portadora de una leiomiomatosis cuyo último embarazo termina por ectópico roto. Las de mayor edad -74 y 75 años- fueron intervenidas una por cistoadenoma seroso del ovario y la segunda por adenocarcinoma de endometrio con miomas intersticiales, subserosos, submucosos y útero bicorne.

* Prof. Asistente. Dpto. Obst. y Ginecología. Facultad de Medicina. Cartagena. 


\section{Menarquia}

En 181 historias encontramos los respectivos datos que nos permiten las siguientes anotaciones: en el $93.3 \%$ de los casos la aparición de la primera hemorragia menstrual cursa entre los 11 y 16 años, edades límites aceptadas como normales (1).
En el cuadro № 2 comparamos las edades de menarquia y respectivos porcentajes entre el grupo de miomatosas y otro testigo proveniente de la $\mathrm{Cl}$. de Maternidad de Cartagena, dos muestras de las mismas condiciones socio-culturales y económicas.

\section{CUADRO № 2}

MENARQUIA : 181 MIOMATOSAS - 298 TESTIGOS

\begin{tabular}{|c|c|c|c|c|c|c|c|c|c|c|}
\hline $\begin{array}{l}181 \\
N^{0}\end{array}$ & $\begin{array}{l}\text { mio } \\
\text { de ca }\end{array}$ & $\begin{array}{l}\text { natosas } \\
\text { os }\end{array}$ & & $\%$ & dad c & $\begin{array}{l}\text { de Menarquia } \\
\text { Edades }\end{array}$ & & $\%$ & $\begin{array}{l}298 \\
N^{0}\end{array}$ & $\begin{array}{l}8 \text { testigos } \\
\text { de casos }\end{array}$ \\
\hline & 9 & & & 5 & & $10-11$ & & 4.7 & & 14 \\
\hline & 32 & & & 17.7 & & 12 & & 21 & & 63 \\
\hline & 41 & & & 22.6 & & 13 & & 27.5 & & 82 \\
\hline & 46 & & & 25.4 & & 14 & & 21 & & 63 \\
\hline & 29 & & & 6 & & 15 & & 16.8 & & 49 \\
\hline & 12 & & & 6.6 & & 16 & & 6 & & 18 \\
\hline & 12 & & & 6.6 & & $17-19$ & & 3 & & 9 \\
\hline & & Edad & Ginecológica & Promedio: & 13.5 & - & Edad & Ginecológica & Promedio & \\
\hline
\end{tabular}

La menor edad ocurrió entre las testigos: un caso a los 10 años. Los porcentajes entre los dos grupos hasta la edad de 16 años -93\% y $97 \%$ - así como la edad ginecológica promedio para ambos -13.5 años- son similares a los índices apuntados para mujeres de Cali y Popayán (2-3).

\section{Relaciones sexuales}

Como es de frecuente ocurrencia en este medio, la mujer de baja cla- se social, por influencia del ambiente familiar y por razones económicas, inicia a temprana edad sus relaciones sexuales. Así encontramos en el grupo testigo utilizado para la menarquia, que la vida sexual se inicia en el $67.9 \%$ entre los 12 a 18 años, es decir, en el curso de la adolescencia; mientras que el $27.4 \%$ lo hace de los 19 a 23 años. Entre las miomatosas la etapa genital activa se inicia entre los 12 a 18 años en el $54.5 \%$ y de 19 a 23 en el $27.9 \%$.

CUADRO NN 3

EDAD DEL PRIMER COITO

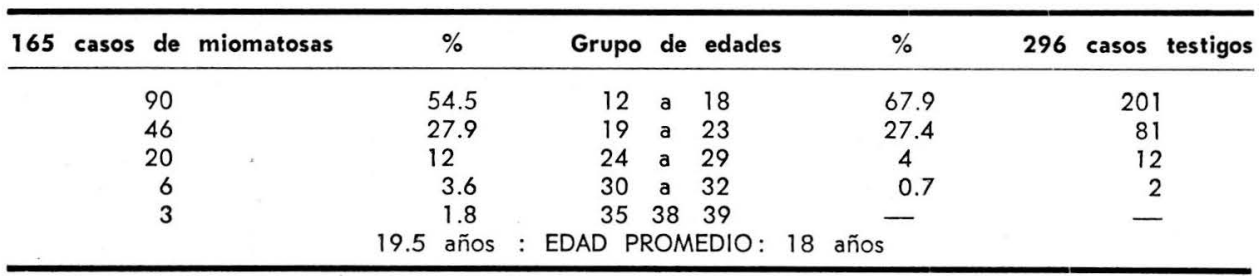


No obstante una misma edad ginecológica promedio (4) de 13.5 años, hay manifiesta diferencia entre los dos grupos en cuanto al comienzo de la actividad genital hasta los 23 años; $12.9 \%$ más tardía entre las miomatosas. Las edades promedio de iniciación de la vida sexual 19.5 y 18 años es similar a la registrada entre las mujeres de Cali según G. Llanos (2).

En relación con el aparte que comentamos, encontramos 14 mujeres vírgenes sobre 178 pacientes, para un porcentaje de 7.9. Sus edades fluctuaban entre los 27 y 48 años. Portadoras de grandes o múltiples miomas, cuya sintomatología obligó a la histerectomía.

Otros aspectos sexuales son los concernientes al orgasmo. En 101 historias se consignaron datos acerca del particular: 77 señoras presentaban orgasmo normal (76.2\%), en 18 la satisfacción sexual era esporádica $(17.9 \%), 5$ eran frígidas: una con dispareunia y 3 con repulsión sexual. Estas mínimas cifras de trastornos sexuales muy probablemente nada tienen que ver con la patología tumoral; ya que se reconoce en ellos una marcada influencia psicosomática derivada de la propia vida conyugal. Por lo demás, esta misma sintomatología es mucho más común en mujeres ajenas a la lesión miomatosa, como es frecuente descubrir en las encuestas de los centros de Planificación Familiar (3-5).

La etapa de convivencia marital fue vivida con más de un marido en el $42.1 \%$ de los casos, fenómeno muy repetido en este tipo social.

\section{CUADRO NN 4}

NUMERO DE CONYUGES EN 154 MIOMATOSAS

\begin{tabular}{ccc}
\hline Niaridos & No de casos & Porcentajes \\
\hline 1 & 89 & 57.9 \\
2 & 43 & 27.9 \\
3 & 17 & 11 \\
4 & 5 & 3.2 \\
1.6 Promedio de maridos por mujer \\
\hline
\end{tabular}

Los anteriores datos dan un total cle 246 maridos con un promedio de 1.6 .

Los años de vida marital fértiles computados a partir del primer coito hasta la fecha de operación, teniendo en cuenta la menopausia (para no tomar en cuenta los años más allá de este límite para las que seguían manteniendo actividad sexual) último coito, etc. suman un total de 3.118 años para 163 mujeres con una duración promedio de 19 años. Con los anteriores datos dejamos establecido en este grupo de miomatosas su gran actividad genital que contrasta con la alta frecuencia de esterilidad que le acompaña.

\section{Gravidez - Paridad - Aborto}

Es motivo de interés las relaciones entre la miomatosis y la función de reproducción femenina. Para muchas mujeres estériles, el problema se reduce a la presencia de nódulos cuya extirpación va seguida de embarazo (11), en algunas estadístiacs hasta el 25 y $40 \%$ (1-6). Otras, por largo tiempo con grandes o múltiples miomas, ven aparecer el embarazo que puede transcurrir hasta el parto; pero en muchos casos su final es el aborto. En el análisis de este acápite, hemos tenido en cuenta lo anotado para el cómputo de los años de vida marital. Se desecharon las historias en las cuales faltaron datos sobre las gestaciones; y las que pre- 
sentaron lesiones de las trompas extirpadas, según protocolo de anatomía patológica. Como es natural, las pacientes vírgenes no fueron incluídas.

De esta manera, sobre un total de 142 casos, encontramos 74 de infertilidad, $52.1 \%, 20$ de ellas primaria $(27 \%)$ y 54 de tipo secundario $(73 \%)$, cifras relativamente altas que dan importancia a la miomatosis en la génesis del problema.

Descontados los 20 casos de infertilidad primaria, nos queda un grupo de 122 pacientes en el cual veremos lo concerniente a gestaciones, paridad, aborto, detallándolas según la edad en que se inició la vida marital y en comparación con el mismo grupo que nos ha servido de testigo antes.

\section{CUADRO N: 5}

GRAVIDEZ, PARIDAD, ABORTOS Y AÑOS DE EXPOSICION EN 122 MIOMATOSAS

\begin{tabular}{|c|c|c|c|c|c|c|c|c|c|}
\hline \multirow[b]{2}{*}{ Iniciación } & \multirow[b]{2}{*}{ de } & \multirow[b]{2}{*}{ vida } & \multirow[b]{2}{*}{ a marital } & \multirow[b]{2}{*}{$N^{0}$} & \multirow[b]{2}{*}{ casos } & \multicolumn{3}{|c|}{ Totales de } & \multirow[b]{2}{*}{ Años Exp. } \\
\hline & & & & & & G. & P. & A. & \\
\hline 14 & a & $19 \quad$ & años & & 76 & 384 & 296 & 88 & 1.697 \\
\hline 20 & a & 25 & " & & 34 & 159 & 116 & 43 & 357 \\
\hline 27 & a & 32 & $"$ & & 10 & 22 & 16 & 6 & 102 \\
\hline 35 & a & 38 & " & & 2 & 1 & 0 & 1 & 6 \\
\hline Totales & & & & & 122 & 566 & 428 & 138 & 2.162 \\
\hline
\end{tabular}

Promediando los datos expuestos en el Cuadro № 5 vemos que las 122 miomatosas en 2.162 años exposición tuvieron 4.6 embarazos por mujer, 1 gestación cada 3.8 años y 1 parto cada 5.05 años. El promedio de abortos fue $24.4 \%$.

\section{CUADRO NN 6}

GRAVIDEZ, PARIDAD, ABORTOS Y AÑOS DE EXPOSICION EN 145 TESTIGOS NO MIOMATOSAS

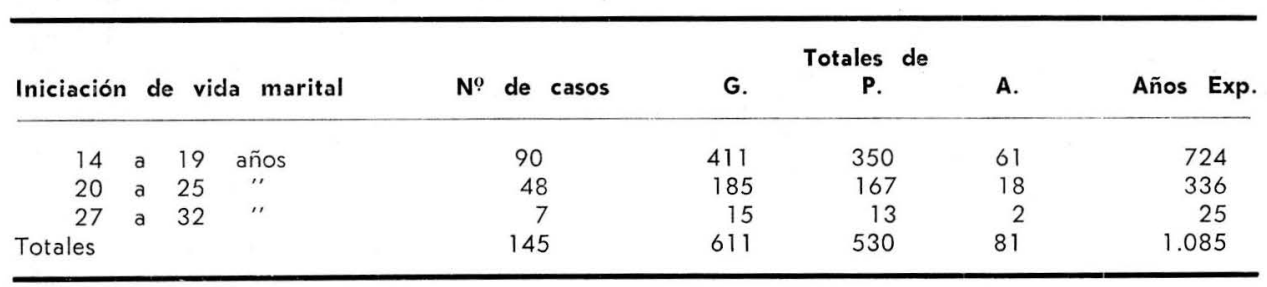

Las cifras del Cuadro № 6 nos ofrecen los siguientes promedios: 145 mujeres en 1.085 años exposición se embarazaron 611 veces, $4.2 \quad 13.2 \%$. gestaciones por mujer; 1 por cada 1.8 años y 1 parto cada 2.04 años. La frecuencia del aborto fue de 


\title{
CUADRO NN 7
}

\begin{abstract}
COMPARACION DE PROMEDIOS DE AÑOS EXPOSICION, AÑOS GRAVIDEZ, AÑOS PARIDAD Y GRAVIDEZ POR MUJER
\end{abstract}

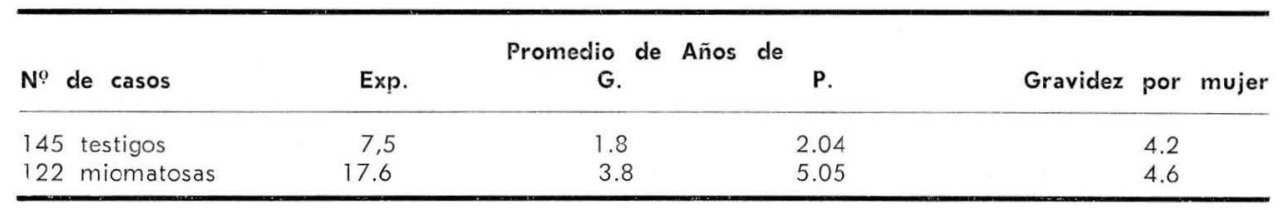

Dados los años de exposición y los promedios anotados, resulta la fertilidad de la mujer miomatosa disminuída en un 50\%, es decir, 2 veces menor que en el grupo testigo, sin tener en cuenta los 20 casos de infertilidad primaria.

Ciertamente, la mujer actualmente intervenida por miomas puede ser una gran multípara. Cursando dicha entidad clínicamente entre la $3^{\text {a }}$ y $4^{\text {a }}$. década de la vida, es lógico que habrá mayor oportunidad de embarazarse cuanto más tempranamente se inicie la vida marital. Encontramos 21 grandes multíparas en el grupo que iniciaron vida sexual entre los 14 y 19 años ( $G .7$ a G.19) y 6 más entre aquellas cuya vida genital comenzó de 20 a 25 años (G.8 a G.17). Algunas de estas mujeres fueron operadas por prolapso uterino; siendo hallazgo de patología los nódulos miomatosos. A partir de los 25 años, disminuye notablemente la fecundidad de las miomatosas. (Ver cuadros 5 y 6. Comparar grupos de edades).

\section{CUADRO № 8}

TASAS COMPARATIVAS DE ABORTO

\begin{tabular}{lccc}
\hline $\begin{array}{l}\text { Iniciación de } \\
\text { vida marital en }\end{array}$ & $\begin{array}{r}\mathbf{1 4 5} \text { de abortos } \\
\text { testigos }\end{array}$ & $\begin{array}{c}\text { sobre embarazos } \\
\mathbf{1 2 2}\end{array}$ \\
miomatosas
\end{tabular}

En relación con el aborto, vemos como es de mayor ocurrencia entre las miomatosas. Si consideramos la tasa biológica del aborto espontáneo entre el 10 y $13 \%$ (7), aparece el grupo control entre límites normales, 13.3; en tanto que para las miomatosas el índice de aborto se eleva al $24.4 \%$.

\section{Esterilidad}

El material de este capítulo ha sido revisado cumpliendo ciertos requisitos. La infertilidad primaria la consideramos por encima de los 4 años de vida marital sin embarazo. Para la secundaria extendemos este lapso a los 6 años después del último par-

\section{CUADRO № 9}

INFERTILIDAD PRIMARIA EN 20 MIOMATOSAS

\begin{tabular}{cccccc}
\hline Iniciación de vida marital & $N^{0}$ de casos & $N^{0}$ de maridos & Años de Exposición \\
\hline 15 a 19 años & 12 & 23 & 205 \\
20 a 25 años & 8 & 10 & 143 \\
Promedios & & - & 1.65 & 17.4 \\
\hline
\end{tabular}


to o aborto. Los casos con lesiones comprobadas de las trompas por estudio histológico, no fueron computados.

En resumen, 20 miomatosas que a edades fértiles inician su vida marital; con 1.65 cónyuge promedio y 17.4 años exposición, no consiguieron un solo embarazo.

La sola localización de los nódulos miomatosos no es causa suficiente para esta esterilidad primaria. Su situación más frecuente fue intramural, en 17 casos: 4 en forma exclusiva, en 7 acompañada de núcleos subserosos, 2 con núcleos submucosos y en 4 más con ambos tipos de localización.

Hubo 1 caso subseroso - submucoso; y 2 más subserosos pediculados, grandes tumores con degeneración mucoide y extensos focos de necrosis, respectivamente. Tampoco el volumen del útero y tumores miomatosos parece tener importancia. El volumen uterino así considerado, varió entre $160 \mathrm{cc}$ y $210 \mathrm{cc}$. los más pequeños; hasta 2.592 y 2.992 cc. los más voluminosos. El volumen máximo del útero de una nulípara, dado por Eastman es de $80 \mathrm{cc}$. (7).

En algunos casos el miometrio alcanzó un espesor hasta de 5 ctms.

\section{CUADRO NN 10}

\section{INFERTILIDAD SECUNDARIA - 54 CASOS}

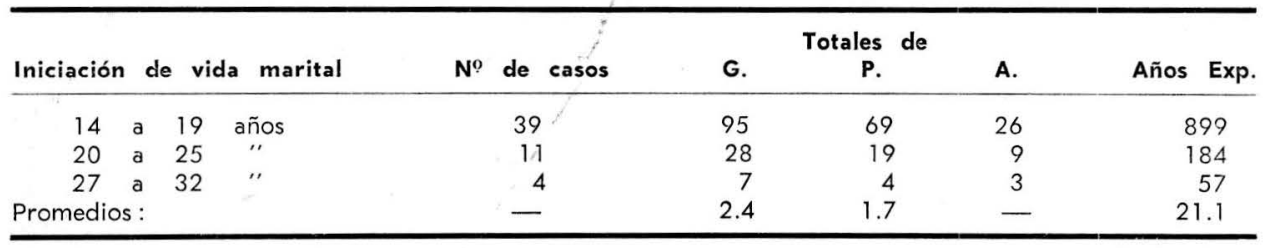

\section{CUADRO N: 11}

\section{INFERTILIDAD SECUNDARIA 54 CASOS}

\begin{tabular}{crc}
\hline $\begin{array}{c}\text { Número de } \\
\text { No de maridos }\end{array}$ & $\begin{array}{c}\text { maridos } \\
\mathbf{N}^{\mathbf{9}} \text { de casos }\end{array}$ & Total \\
\hline 1 & 26 & 26 \\
2 & 16 & 32 \\
3 & 9 & 27 \\
4 & 3 & 12 \\
Total : & 54 & 97 \\
\hline
\end{tabular}

Resumiendo, 54 miomatosos estériles secundarias con 97 maridos en 1.140 exposición se embarazaron 130 veces, 2.4 veces por mujer en 21.1 años promedio; 1 gestación cada 8.8 años y 1 parto cada 12.4 años de vida marital. La frecuencia del aborto se eleva en este grupo al $29 \%$.

La mayor multiparidad registrada fue de $G .7$ para 6 abortos y un embarazo ectópico. Dos pacientes más tuvieron 5 embarazos cada una para 5 partos y 4 abortos respectivamente.

\section{Localizaciones miomatosas}

La situación y desarrollo de los núcleos miomatosos hace que ordinariamente el útero sea de contorno irregular, de superficie nodular y la mayoría de las veces notablemente aumentado de volumen ocn un espesor considerable. 
CUADRO N: 12

LOCALIZACION DE NODULOS MIOMATOSOS - 185 CASOS

\begin{tabular}{lrrrrrrr}
\hline Localizaciones & IM. & IM. SS. & IM. SS. SM. & IM. SM. & SS. & SM. & SM. SS. \\
\hline No de casos & 90 & 42 & 19 & 14 & 9 & 8 & 3 \\
Porcentajes & 48.6 & 22.7 & 10.3 & 7.6 & 4.9 & 4.3 & 1.6 \\
\hline
\end{tabular}

Observamos que la situación más frecuente es la intramural que individualmente ocupa el $48.6 \%$ y en combinación con las otras localizaciones llega hasta el $89.2 \%$.

\section{CUADRO N: 13}

VOLUMEN UTERINO EN 167 CASOS

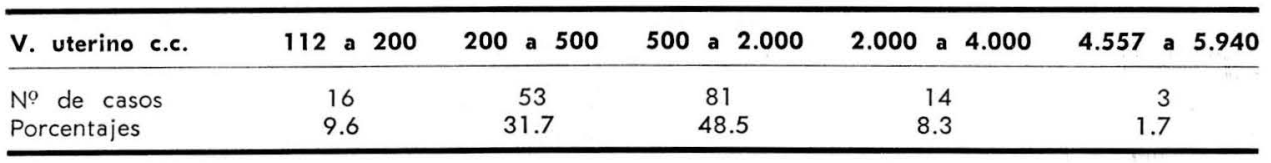

Según Eastman (7) el útero de una mujer multípara tiene un volumen aproximado a los 200 cc. límite dentro del cual se encuentra el $9.6 \%$ de nuestros casos. El $80.2 \%$ va entre los 200 cc. y 2.000 cc. Algunas piezas correspondieron a mujeres vírgenes y nuligestantes con un gran volumen ( 3.570 a 3.942 cc.). El espesor del miometrio varió de 1 a 3 cmts. en 35 casos; de 3.5 a $7 \mathrm{cmts}$. en 20 casos. En una paciente alcanzó 12.5 cmts.

\section{Endometrio}

En 177 casos $94.1 \%$ se obtuvo estudio histológico del endometrio de los úteros extirpados, con los resultados compilados en el Cuadro $\mathrm{N}$ ? 14.

\section{CUADRO № 14}

ENDOMETRIO EN 177 CASOS DE MIOMATOSIS

\begin{tabular}{|c|c|c|}
\hline Tipo & $N^{0}$ de casos & Porcentaje \\
\hline Proliferativo & 117 & 66.1 \\
\hline Hiperplásico & 15 & 8.4 \\
\hline Secretorio & 25 & 14.1 \\
\hline Atrófico & 17. & 9.6 \\
\hline Otros & 3 & 1.7 \\
\hline
\end{tabular}

Como se ve la mayor frecuencia fue de endometrio proliferativo ( $66.1 \%$ ) que junto con el hiperplásico constituyen el $80.2 \%$ de todos los casos. Los últimos 3 corresponden a dos endometrios con autólisis $y$ uno en estado de reposo.

\section{Endometrio Proliferativo}

Es de interés relacionar el tipo de endometrio con el día del ciclo menstrual y la presencia o no de cuerpo lúteo en el ovario. A este respecto, del análisis de 82 mujeres en edades fértiles entre los 24 y 48 años con endometrio proliferativo, se obtuvieron los siguientes datos agrupados en el Cuadro № 15.

CUADRO N: 15

ENDOMETRIO PROLIFERATIVO - DIA DEL CICLO CUERPO LUTEO 82 CASOS

Día del ciclo $\begin{gathered}\text { Cuerpo Lúteo } N^{0} \text { de casos } \\ \text { Presente No presente }\end{gathered}$

\begin{tabular}{|c|c|c|c|}
\hline 6 a 12 & & 17 & 17 \\
\hline 10 a 14 & 8 & - & 8 \\
\hline 14 a 15 & - & 4 & 4 \\
\hline 16 a 30 & 13 & 34 & 47 \\
\hline 33 a 37 & 2 & 2 & 4 \\
\hline ? & 2 & - & 2 \\
\hline Totales & 25 & 57 & 82 \\
\hline
\end{tabular}


En 25 casos la histología de los ovarios confirmó un cuerpo lúteo en correspondencia con el día del ciclo. En los restantes 57, en los cuales los ovarios no fueron extirpados o bien no se encontró cuerpo lúteo en las piezas examinadas, las relaciones entre el día del ciclo y endometrio, fue como sigue: en 17 casos - por debajo de los 12 días- había correspondencia con la fase proliferativa; en 40 no se correspondían ya que la fecha del ciclo menstrual indicaba fase progestacional.

En resumen, solo en 17 casos $20.7 \%$ del total de 82 , se correlacionan el endometrio proliferativo y el día del ciclo. Los otros 65 constituídos por los 25 con cuerpos lúteos y 40 que señalan fase progestacional, no están de acuerdo con el estado endometrial $(79.3 \%)$. Esta falta de concordancia sería uno de los factores de la infertilidad de las miomatosas. En un momento dado de la evolución de la enfermedad, el endometrio se comporta como uno de tipo basal, inmaduro, que no responde a la acción de la progesterona, bloqueando así una presunta nidación.

\section{Endometrio Hiperplásico}

Esta condición estuvo presente en 15 casos. Tres eran seudoquísticos seniles en menopáusicas. Los restantes 12 , en mujeres entre los 29 y 45 años. Los ovarios fueron extirpados en 5 casos. Los detalles más salientes los destacamos en el Cuadro $\mathrm{N}$ ? 16.

\section{Endometrio Secretorio}

(Cuadro № 17). Se encontró en 25 casos. Una mujer era menopáusica. 12 estaban entre los 30 y 39 años. 11 entre los 40 y 48 años. Una más tenía 50. En 13 pacientes se practicó ovariectomía. 12 ovarios presentaban cuerpo lúteo.
Rev. Col. Obst. y Ginec.

\section{CUADRO NN 16}

presente No presente

\section{ENDOMETRIO HIPERPLASICO. - 15 CASOS}

\begin{tabular}{|c|c|c|c|c|}
\hline Día del ciclo & $\begin{array}{l}\text { Cuerpo } \\
\text { presente No }\end{array}$ & $\begin{array}{l}\text { Lúteo } \\
0 \text { presente }\end{array}$ & $\mathbf{N}^{0}$ & de casos \\
\hline 5 a 7 & - & 2 & & 2 \\
\hline 17 a 30 & 1 & - & & 5 \\
\hline 34 a 35 & - & - & & 2 \\
\hline ? & - & 1 & & 3 \\
\hline Menopáusicas & - & 1 & & 3 \\
\hline Totales & 1 & 4 & & 15 \\
\hline
\end{tabular}

CUADRO N: 17

ENDOMETRIO SECRETORIO. - 25 CASOS

\begin{tabular}{cccc}
\hline Día del ciclo & \multicolumn{2}{c}{ Cuerpo Lúteo } & No de casos \\
\hline 7 & - & - & 1 \\
12 a 13 & 1 & - & 2 \\
14 a 30 & 7 & - & 12 \\
32 a 36 & 1 & - & 3 \\
$?$ & 2 & 1 & 6 \\
Menopáusicas & 1 & - & 1 \\
Totales & 12 & 1 & 25 \\
\hline
\end{tabular}

\section{Endometrio atrófico}

Esta lesión endometrial se halló en 17 casos. Junto a los dos con autólisis constituye el $10.7 \%$ del total de 177 pacientes que hemos estudiado. Dos eran menopáusicas. Cuatro tenían entre 50 y 57 años. Las restantes entre los 36 y 49 años. Los datos incompletos no nos permiten presentarlos en cuadro como con los anteriores. En dos casos se informó de cuerpos lúteos al examen histológico.

En resumen, 25 endometrios en fase secretora, 25 cuerpos lúteos en los casos con endometrio proliferativo y 4 más en los de hiperplasia y atrofia, hablan de la alta frecuencia ovulatoria de las miomatosas y consiguientemente de sus posibilidades de fecundación. Representan el 50.5\% de todas las pacientes en edad fértil 
comprendidas en este capítulo, cuyos ciclos ováricos bifásicos permitirían ser fecundadas; en tanto que la condición endometrial adversa y la localización de los nódulos miomatosos pudieran interferir con la nidación del huevo o con el curso del embarazo.

\section{Patología de Ovario}

El ovario como fuente de estrógeno ha sido considerado parte principal en la producción de la miomatosis uterina, a tal punto que algunos autores han señalado que esta entidad debiera ser discutida entre las discrinias ováricas (8). De aquí, que apuntemos algunos datos sobre su patología en el presente estudio.

En el tratamiento dado a la miomatosis uterina, la gónada fue intervenida en forma total o parcial, uni - bilateralmente, en 111 casos. De su examen histológico resultó 33 veces normal, $29.7 \%$. La patología de los restantes 78 casos $(69.3 \%)$ la consignamos en el siguiente cuadro.

\section{CUADRO № 18}

PATOLOGIA DE OVARIO - 78 CASOS

\begin{tabular}{lcc}
\hline Patología & No de casos & Porcentaje \\
\hline $\begin{array}{l}\text { Ovarios poliquísticos } \\
\text { Quistes simples y }\end{array}$ & 47 & $60.25 \%$ \\
$\quad$ hemorrágicos & 14 & 17.9 \\
Quistes luteínicos & 3 & \\
$\quad$ hemorrágicos & 3 & \\
$\begin{array}{l}\text { Cistadenoma seroso } \\
\text { Cáncer de ovario }\end{array}$ & 2 & \\
Tumor de Brenner & 1 & \\
Tumor Dermoide & 3 & \\
Endometriosis ovárica & 2 & \\
Procesos infecciosos & 3 & \\
crónicos & & \\
\hline
\end{tabular}

Los ovarios poliquísticos, los de mayor frecuencia, estaban constituí- dos por pequeños, múltiples quistes uni o bilaterales. Los dos Ca. de ovarios: un adenocarcinoma con extensión a trompa izquierda y un Cistoadenocarcinoma papilar con posterior invasión a cúpula vaginal.

\section{Síntomas}

Muy de acuerdo con lo que señalan los textos sobre el particular, las molestias más frecuentemente referidas fueron en su orden: dolor en hipogastrio, en ocasiones cólico, a veces con irradiación a región sacro lumbar, generalmente de tipo gravativo, acompañando a una masa en bajo vientre. La tumoración se describió al examen como dura, irregular, nodular, ocasionalmente dolorosa. En segundo lugar, aparecen los trastornos del ciclo menstrual, consistentes en menometrorragias, en algunos casos con coágulos y frecuentemente con dismenorrea. Otros disturbios lo fueron: polimenorrea, amenorrea (4 veces), leucorrea, particularmente en los miomas submucosos pediculados. Otras pocas mujeres acusaron dispareumia, molestias a la micción, peso en genitales, etc.

\section{Patología asociada}

El hecho mismo de la larga duración, y época de la vida en que se manifiesta la miomatosis uterina, facilita su asociación con la más diversa patología. Queremos sin embargo, llamar la atención hacia las lesiones cancerosas en particular, y registraremos, de paso, las otras entidades encontradas.

En 188 casos hubo 12 procesos malignos, distribuídos en el Cuadro N. $19(6.4 \%)$.

No obstante la búsqueda sistemática del $\mathrm{Ca}$. in situ del cérvix entre las mujeres asistentes al Programa 
CUADRO N: 19

MIOMAS $Y$ LESIONES MALIGNAS - 12 CASOS

\begin{tabular}{llrr}
\hline Localización & Tipo & No de casos \\
\hline Cérvix & Ca. in situ & 5 & 2.7 \\
& Ca. Epidermoide & 1 & 0.53 \\
Cuerpo Uterino & Leiomiosarcoma & 2 & 1.06 \\
Endometrio & Adenocarcinoma & 2 & 1.06 \\
Ovario & Adenocarcinoma papilar & & \\
& Cistoadenocarc. papilar & 1.06 \\
\hline
\end{tabular}

de Planificación Familiar de Cartagena, solo se han conseguido 3 entre 700 señoras (hasta diciembre de 1966). 1 por cada 233 personas. $0.43 \%$. Por el contrario, en 166 miomatosas en edad fértil, hemos encontrado 5; 1 por cada 33.2 mujeres. Un porcentaje de 3 , es decir, 7 veces más frecuente en este grupo. Totalizando las lesiones malignas, resulta 1 por cada 15.6 mujer miomatosa. Sería de desear otras observaciones para controlar esta información. Por lo pronto, dichas cifras indicarían la necesidad de un tratamiento menos conservador o una vigilancia más de cerca para la miomatosis uterina.

\section{Otra patología}

En el Cuadro № 20 detallamos otros procesos patológicos y enfermedades que tuvieron lugar en el grupo de miomatosas.

\begin{tabular}{|c|c|c|c|}
\hline \begin{tabular}{ccc} 
& \multicolumn{2}{c}{ CUADRO } \\
TRA & PATOLOGIA EN
\end{tabular} & $\begin{array}{l}\text { No: } \\
188\end{array}$ & & IATOSA \\
\hline & & $\begin{array}{l}N^{0} \text { de } \\
\text { casos }\end{array}$ & Porcentaje \\
\hline Pólipos Endometriales & 7 & & \\
\hline Pólipos Endocervicales & 11 & 21 & 11.2 \\
\hline Ambas localizaciones & 3 & & \\
\hline Adenomiosis & & 5 & 2.65 \\
\hline $\begin{array}{l}\text { Fibroadenoma de la } \\
\text { mama }\end{array}$ & 2 & & \\
\hline Quiste de la mama & 1 & 3 & 1.6 \\
\hline Embarazo Ectópico & & 5 & 2.65 \\
\hline Bocio Nodular no Tóxico & & 5 & 2.65 \\
\hline Obesidad & & 25 & 13.3 \\
\hline Hipertensión Arterial & & 10 & 5.3 \\
\hline Colelitiasis & & 5 & 2.65 \\
\hline Totales: & & 79 & 42.0 \\
\hline
\end{tabular}

En la esfera genital se destaca la poliposis endometrio-cervical y la adenomiosis. La primera con 21 casos para un $11.2 \%$. En su artículo sobre Endometriosis, Amaya León y col. apuntaron una relación del 53\% entre adenomiosis y fibromiomas uterinos (9). En el presente trabajo representa el $2.65 \%$. El endometrio de la adenomiosis, de tipo inmaduro, inactivo, la mayoría de las veces, similar al de los pólipos, pudiera ser uno mismo con el de características basales, proliferativo e hiperplásico por la excesiva y persistente estimulación estrogénica, que hemos encontrado en el $80.2 \%$ de los úteros miomatosos.

\section{Gestación ectópica}

Cuatro pacientes relataron entre sus antecedentes sendos embarazos ectópicos. Una más fué intervenida por ectópico roto. G.4 P.O A.3. Presentó focos de hialinización y necrosis de nódulos intramurales. La frecuencia esperada del ectópico va de 0.3 a $0.5 \%$ (10) (12). En el grupo estudiado se eleva al $2.65 \%$ lo que destaca el papel de la miomatosis como predisponente.

\section{Obesidad}

No vamos a catalogar esta condición, frecuentemente acompañando la miomatosis, como estado patológico. Representó el $13.3 \%$ aun cuando este aspecto somático no se inscribe rutinariamente en las historias. 
Pudiera indicar la presencia de un metabolismo distorsionado en estas pacientes.

\section{Bocio nodular no tóxico}

5 mujeres fueron intervenidas por esta causa, $2.65 \%$. Ninguna presentó otra asociación patológica.

\section{Hipertensión arterial}

10 pacientes, $5.3 \%$ fueron consideradas por el Departamento de Medicina Interna como hipertensas. Dos con insuficiencia cardíaca congestiva. Una de ellas diabética. Tres, eran obesas; una de las cuales era también colelitiásica.

\section{Colelitiasis}

Cinco casos para un $2.65 \%$. Una más presentó Agenesia de la vesícula biliar.

\section{RESUMEN}

Se presentan las observaciones recogidas en el estudio de 188 casos de miomatosis uterina con certificación de anatomía patológica. Se destacan los siguientes hechos: la disminución de fertilidad de las miomatosas, su esterilidad y alta frecuencia de aborto. La persistencia del endometrio de tipo proliferativo en el $66.1 \%$ e hiperplásico $14.1 \%$ en los úteros extirpados; sin correspondencia en la gran mayoría de las veces con la edad del ciclo menstrual y la presencia de cuerpo lúteo en el ovario. Se sugiere que pudiera ser esta discordancia causa de importancia en la infertilidad de las miomatosas.

Por último, se señala el porcentaje de $\mathrm{Ca}$. in situ del cérvix, 7 veces más alto que en mujeres testigos; y el hecho de haber encontrado 1 lesión maligna por cada 15.6 mujeres.

\section{BIBLIOGRAFIA}

1 NOVAK E. R.: Tratado de Ginecología. 1ạ Edición española. pág. 81. Edit. Interamericana S. A. 1965.

2 LLANOS G.: Epidemiología del Aborto en Cali. Vol. $2^{\circ}$ Sem. sobre Demografía Asoc. Col. Fac. Med. Oct. 1965.

3 ERAZO J. V.: Encuesta de Opinión sobre Planificación Familiar en los Municipios de Popayán y Timbío. Bol. del $2^{\circ}$ Sem. sobre Demografía. Asoc. Col. Fac. Med. Oct. 1965 .

4 BUXTON and ENGLE, "cit. por Hartzman C. G.: Science and the Safe Period. Edit. The Williams \& Wilkins Company. Baltimore 1962

5 AGUIRRE CASTAÑO A.: Algunas bases epidemiológicas de la Mortalidad Infantil. Publicación de la Fac. de Med. Univ. del Valle 1966.

6 BERNAL H. E.: Esterilidad primaria de origen miomatoso curada por miomectoma. Rev. Col. Obst. Gin. Vol. VII. No 31956.

7 EASTMAN N. J.: Obstetricia de Williams $3^{a}$ edit. española. H. Vela Triviño traductor. Edit. UTEHA. México pág. 32.

8 CONILL MONTOBBIO V.: Tratado de Ginecología y de Técnica Terapéutica Ginecológica. 2a Edic. Edit. Labor S. A. 1950. pág. 542.

9 AMAYA LEON H. y col.: Endometriosis. Rev. Col. Obst. Gin. Vol. XI. № 3. 1960.

10 DI PAOLA G. y col. Evolución del Embarazo en las mujeres tratadas por infertilidad. Rev. Col. Obst. Gin. Vol. VI Ne 1. 1954.

11 ECHEVERRY $V$. H.: Cinco casos de miomectomía durante el embarazo. Rev. Col. Obst. Gin. Vol. V No 41954.

12 GOMEZ PALACINO J. A.: Noventa y cinco casos de embarazo ectópico. Rev. Col. Obst. Gin. Vol. V. No 4. 1954. 